\title{
Boundary effects in a quasi-two-dimensional driven granular fluid
}

\author{
N. D. Smith and M. I. Smith* \\ School of Physics and Astronomy, University of Nottingham, Nottingham NG7 2RD, United Kingdom
}

(Received 14 September 2017; published 29 December 2017)

\begin{abstract}
The effect of a confining boundary on the spatial variations in granular temperature of a driven quasi-twodimensional layer of particles is investigated experimentally. The radial drop in the relative granular temperature $\Delta T / T$ exhibits a maximum at intermediate particle numbers which coincides with a crossover from kinetic to collisional transport of energy. It is also found that at low particle numbers, the distributions of radial velocities are increasingly asymmetric as one approaches the boundary. The radial and tangential granular temperatures split, and in the tails of the radial velocity distribution there is a higher population of fast moving particles traveling away rather than towards the boundary.
\end{abstract}

DOI: 10.1103/PhysRevE.96.062910

\section{INTRODUCTION}

Granular materials consist of macroscopic particles whose dynamics are not influenced by thermal fluctuations. However, when subjected to external mechanical perturbations, the collective behavior of the particles may exhibit many similarities to conventional phases of matter such as gases, fluids, or solids. For example, a driven "granular gas" may exhibit a velocity probability distribution that is very nearly Maxwellian $P(v) \sim$ $a v \exp \left(-b m v^{2}\right)[1,2]$ (for two dimensions). At higher particle concentrations, phenomena such as liquidlike instabilities [3], crystallization [4], glassy dynamics [5] have all been observed, demonstrating the strength of the analogy between granular and molecular systems. Given these apparent similarities it is common to define an effective "granular temperature" $\left(T_{\mathrm{g}}\right)$ for these athermal systems in terms of the mean kinetic energy per particle $\left(\left\langle v^{2}\right\rangle\right)$, to characterize the fluctuations in particle velocity [2].

However, assessing when the results of steady-state granular systems are equivalent to the behavior of their molecular counterparts requires great care. Dilute granular gases of particles are known to display a rich variety of behaviors not found in thermodynamic equilibrium that arise from the dissipative nature of the collisions between particles. Phenomena such as ring collisions [6], clustering [7], swarming [8], etc., lead to behavior unique to these nonequilibrium systems. The presence of dissipative collisions also gives rise to non-Gaussian velocity distributions $[2,9,10]$. Study of the tails of these velocity distributions has shown that they are found to be overpopulated when compared with a Gaussian distribution of equivalent temperature.

A particularly striking example of the differences between granular and molecular systems is the nature of temperature itself. In equilibrium, temperature is a scalar quantity. However, it has been shown that in the presence of gravity, the vertical component of the granular temperature in the system

*Corresponding author: mike.i.smith@ nottingham.ac.uk

Published by the American Physical Society under the terms of the Creative Commons Attribution 4.0 International license. Further distribution of this work must maintain attribution to the author(s) and the published article's title, journal citation, and DOI. at a particular point can differ from the value measured in the horizontal plane, leading to the counterintuitive concept of a direction dependent temperature [11,12]. Despite this complexity, granular temperature provides a useful means of characterizing spatial variations in the particle energy.

A common realization of a driven granular fluid is a quasi-two-dimensional (2D) setup in which particle motions are excited by either a turbulent flow of gas [8], moving walls [13], or a rough, vibrating plate $[14,15]$. In each case, the injection of energy into the system excites the motion of the particles, resulting in dissipative collisions between different particles and with the boundaries of the experiment. This leads to a steady state in which the energy input is balanced by losses. A number of studies have attempted to understand how granular temperature is affected by the fluid parameters (coefficient of restitution, particle number density, etc.), and system geometry [2,16-20]. It is also known that boundaries can significantly alter the average dynamics of particles, resulting in gradients in the granular temperature across an experimental cell [20,21].

In studies where a quasi-2D granular fluid is being used as a model hard sphere system to study, by way of analogy, the generic behavior of molecular systems, this spatial variation in temperature could have important consequences. In some experiments at high density this limitation can be mitigated by only analyzing data collected from regions distant from the boundary where the temperature profile is assumed to be flat [4]. However, in experiments examining the effects of, for example, confinement in "glassy" samples [22], these boundary effects cannot be avoided and may represent an important consideration in subsequent analysis. As the particle number density is reduced it is also not clear where in the cell, if at all, the effects of boundaries can be safely discounted.

In this paper we examine the role of a confining wall in a driven quasi-2D granular fluid. Through examining the spatial variations in granular temperature with varying particle area fraction we identify a crossover between two types of behavior as the particle area fraction increases.

\section{EXPERIMENTS}

Our experimental setup consists of a thick horizontal circular aluminum plate that is bordered by a flat sided aluminum ring (internal diameter $225 \mathrm{~mm}$ ). The base plate 
is attached to a very heavy frame by three radial leaf springs, and was carefully leveled by adjusting antivibration feet at the base of the frame. Measurements made using an accelerometer at different locations were the same to within the accuracy of the accelerometer $( \pm 0.05 \mathrm{~g})$. We also carefully observed the vibrations of the plate using a strobe light that had been slightly detuned from the vibrational frequency to look for normal modes and observed none. The final level was checked by ensuring the time averaged radial density profile of 100 particles filmed moving over the plate was independent of the azimuthal direction both before and after experiments. Small vertical vibrations $(f=50 \mathrm{~Hz})$ were excited by an electromagnet situated below the plate (amplitude $\sim 0.25 \mathrm{~mm}$ ). The surface of the aluminum plate was covered by P120 grit sandpaper, onto which between ten and 500 acrylic beads (diameter $=8 \mathrm{~mm}$ ) were placed. The vibrations, coupled with the rough surface, caused the motion of the particles over the surface of the plate confined to a quasi-2D layer. Filming a single particle repeatedly being dropped onto blocks of acrylic and aluminum enabled us to estimate the coefficient of restitution of particle-particle and particle-wall collisions as $e_{\text {particle }}=0.8$ and $e_{\text {wall }}=0.85$, respectively.

Before each experiment, the particles were vibrated for $10 \mathrm{~min}$ to ensure that the system had reached a steady state. The motion of the particles was then filmed with a Panasonic HC-X1000 camera (resolution $1920 \times 1080$, $60 \mathrm{fps}$ ), under strong uniform illumination. For each experiment enough video was acquired to provide at least 2500000 data points (positions of $N$ particles in $M$ frames). Using MATLAB the position of each particle in every frame was measured using a binary threshold followed by a Hough transform. The velocities of each particle were then calculated from their positions in consecutive frames. Particle positions were measured with an uncertainty of $1.5 \%$ of the particle diameter. Using a small number of large particles enables us to measure the position of every particle in every frame with a high degree of accuracy and thus extract the entire velocity statistics (total, radial, and tangential) for each experiment.

To analyze the data we split the cell into seven equal-area rings (see Fig. 1, inset), assigning a particle to a ring based on its centroid in a particular frame. Since it is impossible for the center of mass of a particle to be closer than the particle radius to the boundary we exclude this area from the calculation. This results in the outermost ring having approximately the same width as the particle diameter. Choosing seven rings resulted in a good balance between good statistics in each ring and enough resolution to understand how quantities such as the temperature and volume fraction varied with radius. Errors in the respective granular temperatures were calculated by splitting each data set into ten equal length movies, measuring the granular temperature of these subsets of the data and calculating the subsequent standard deviation (final error $\sim \sigma / 10^{0.5}$ ).

\section{RESULTS AND DISCUSSION}

\section{A. Mean particle area fraction and granular temperature}

Figure 1(a) shows the granular temperature $\left(T_{\mathrm{g}}=\left\langle v^{2}\right\rangle\right)$ at different radial values for different numbers of particles
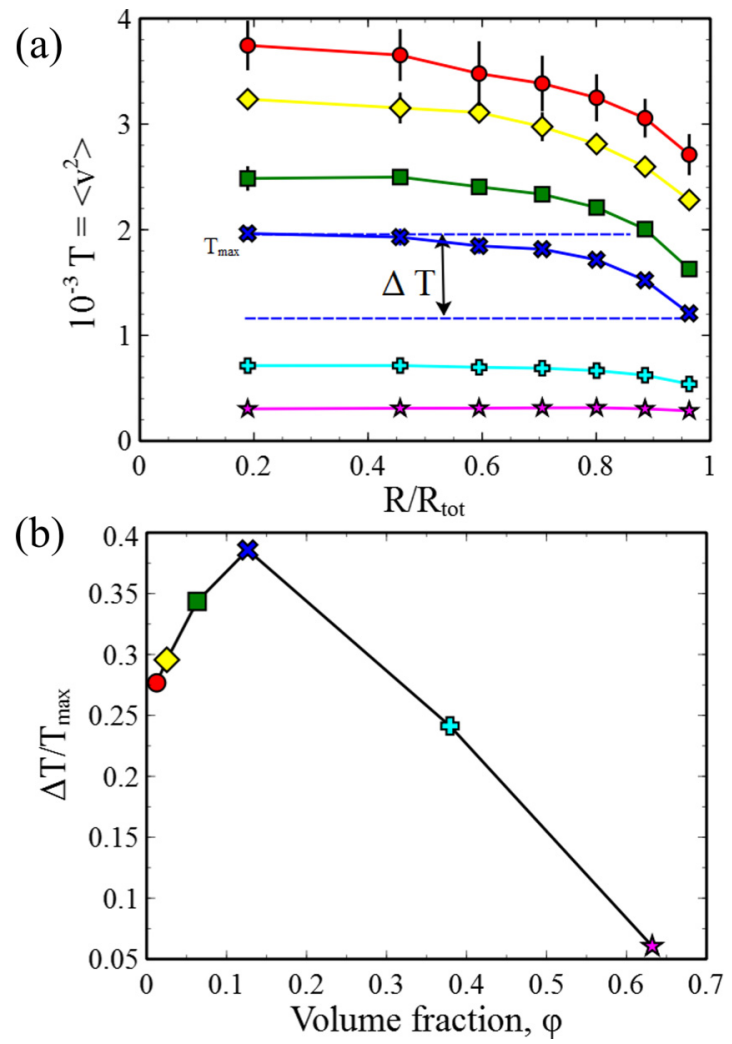

FIG. 1. Effect of a circular boundary on granular temperature

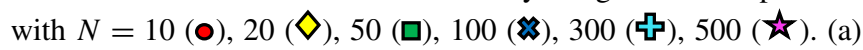
Granular temperature for particles in different radial rings (schematic shown as inset in lower panel). The temperature drops between the center and edge of the experimental cell by an amount $\Delta T$. (b) A plot of $\Delta T / T_{\max }$ for the different particle area fractions. $\Delta T / T_{\max }$ provides an indication of the flatness of the radial temperature profile. The plot shows a peak which appears at intermediate values of the particle area fraction $(N=100)$.

ranging from $10(\bullet)$ up to 500 (star), and corresponding to particle area fractions $\phi \sim 0.013-0.63$. The radial values indicated correspond to the midpoint between the inner and outer boundary of each ring normalized by the radius of the plate.

The granular temperature increases monotonically with decreasing particle number. "Heat" is added to the system through energy injection at the single particle level via contact with the vibrating base and is, to a first approximation, independent of particle number. Under steady-state conditions, this energy input is balanced by dissipation through the inelastic particle-particle or particle-wall collisions. The increasing number of collisions with particle number results in the decreasing steady-state temperature.

Enhanced loss of energy at the edge of the cell results in a radial gradient in temperature [see Fig. 1(a)]. However, the nature of this temperature drop depends sensitively on the details of the system and particle interactions as we discuss in detail below.

In Fig. 1(a), $\Delta \mathrm{T}$ is defined as a way of quantifying the changes in granular temperature between the central ring and the boundary. Given the large changes in mean granular temperature with particle number, we focus on the relative 
temperature drop $\Delta T / T_{\max }$ which gives a measure of the flatness of the temperature profile. This is of relevance to the interpretation of experiments near boundaries at different particle area fractions. Figure 1(b) shows how $\Delta T / T_{\max }$ varies for particle area fraction. Interestingly we observe a peak $\phi \sim$ 0.13 (corresponding to $N=100$ ). This is a counterintuitive result, which implies that the flattest radial temperature profiles are found at both high and low particle area fractions.

Movie 1 in the Supplemental Material [23] shows two short examples of the particle dynamics with $N=20$ and $300(\phi \sim$ $0.025,0.379$ ). The dynamics are qualitatively very different and representative of the behavior observed to the left and right of the peak in Fig. 1(b). At low particle area fractions we observe that particles tend to collect near the boundary, executing short, apparently random walks, before moving on relatively straight trajectories across the tray, with relatively infrequent collisions with other particles ("kinetic transport"). In contrast, at high particle numbers the dynamics is dominated by interparticle collisions, with a much less obvious difference between the motion of particles near the center and boundary of the experimental cell ("collisional transport"). A crossover between kinetic and collisional transport mechanisms has been observed at particle volume fractions $\sim 0.15$ for a granular gas subjected to simple shear in three dimensions [24]. While our system is two dimensional one would expect a similar crossover at comparable particle area fractions.

\section{B. Radial profile in particle area fraction}

The change of behavior either side of a maximum in $\Delta T / T_{\max }$ at $\phi \sim 0.13$. correlates well with changes in the radial profile of the local particle area fraction [see Fig. 2(a)]. The time averaged profile for low particle numbers $(N<100)$ shows a steep rise as the boundary is approached. However, for higher particle numbers there is a much more uniform local particle area fraction. A slight flattening of this profile is first seen for $N=100(\phi \sim 0.13)$.

Why does the relative particle area fraction profile begin to flatten at the values observed? Consider a simple thought experiment in which we take all the particles and push them radially outwards until the particles form a loosely packed ring near the boundary. What is the maximum number of particles that can be located in the outermost analysis ring? Assuming a random loose packing of $\phi=0.775$ [25] this corresponds for our experimental configuration to $N_{c} \sim 88(\langle\phi\rangle \sim 0.11)$ particles. Consequently, at higher numbers of particles there is a decreasing upper bound on the maximum relative particle area fraction that can be achieved (e.g., $N=500, \phi_{\max } /\langle\phi\rangle \sim$ 1.22). While this is still a little higher than the values measured in Fig. 2(a) it is obvious that there will be a large entropic penalty to such an abrupt change in particle density, resulting in a pressure that tends to reduce this maximal value still further. While this is a very crude analysis it agrees with the measured critical value at which the particle density profile begins to flatten out for our experiments. An additional way to understand the changes that occur as a function of particle density is to examine changes in the particles' mean free path. The value of the particle area fraction measured in the outermost ring for $N=100$ is $\phi=0.185$. This corresponds to a mean free path of $12 \mathrm{~mm}$. This size range is important since
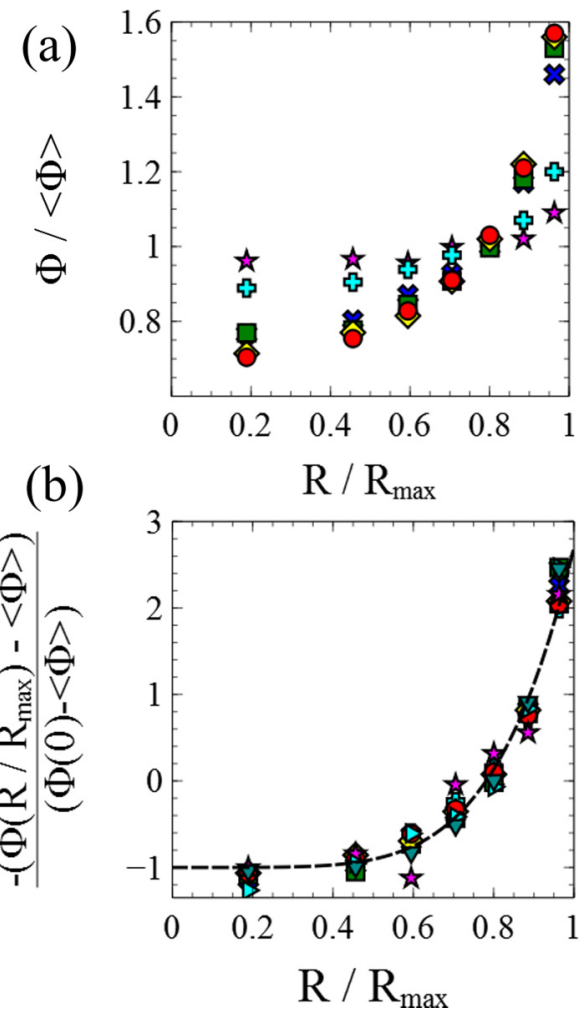

FIG. 2. Particle area fraction profiles. (a) The normalized particle area fraction as a function of radius for $N=10(\mathbf{0}), 20(\diamond), 50(\square)$, 100 (\$), 300 (引), 500 (支). (b) The particle area fraction profiles of all systems, using a simple scaling, collapse onto a single curve which is not affected by increasing the drive amplitude by a factor of $2(\nabla)$ or using brass beads $(\nabla)$ with a different coefficient of restitution.

it is comparable to the particle diameter $(8 \mathrm{~mm})$. Consequently as we move towards $N>N_{\mathrm{c}}$ we move into a regime in which particles near the edge must begin to become caged by surrounding particles; hence "heat" transport via interparticle collisions must begin to become more significant.

Given this clear difference in the mechanism of heat transport, it is surprising that the particle local area fraction curves all bear a similar shape (in contrast with the temperature curves in Fig. 1). In Fig. 2(b) we plot the deviation of the local particle area fraction from its mean value divided by the deviation at $r=0$ (i.e., $-[\phi(r)-\langle\phi\rangle] /[\phi(0)-\langle\phi\rangle])$. To estimate $\phi(0)$ we fit the $\phi(r)$ data points and equate the intercept with $\phi(0)$. All the curves $\phi(r)$, where $r=R / R_{\max }$, are well fitted by an equation of the form $\phi(r)=a\left(r^{b}+c\right)$. Scaling the data in this way we observe a good collapse for all data sets [see Fig. 2(b)]. Substitution of $\phi(r)=a\left(r^{b}+c\right)$ into $-[\phi(r)-\langle\phi\rangle] /[\phi(0)-\langle\phi\rangle]=(b+1) r^{b}-1$. The collapse of the data therefore shows that all the curves obey the same power law exponent $b$.

To explore this further we also plot $\phi(r)$ for experiments conducted at twice the drive amplitude, giving rise to a larger granular temperature. Figure 2(b) shows an example data set for 50 particles $(\boldsymbol{\nabla})$ scaled in the same way. The exponent of the data is unaffected by the granular temperature. Similar tests with 50 brass particles of $8 \mathrm{~mm}$ diameter (different coefficient 
of restitution) also show very similar exponents, confirming the generality of this result.

The change in the particle area fraction profile with increasing bead number [see Fig. 2(a)] helps to explain the peak in $\Delta T / T$ with increasing particle number. In the limit of small particle numbers, the dynamics of particles is affected predominantly by collisions with the walls. Heat is transported across the experimental cell through the momentum of a moving particle. When the particle hits the wall, dissipation cools the particle. It therefore dwells in this region of the experimental cell for a relatively long period of time. On average this results in the local particle area fraction increasing near to the edge of the tray. However, at small enough particle numbers the probability of an interparticle collision remains low. This means that a cold particle can random walk back towards the center of the tray with a relatively high probability. Under these circumstances the returning cold particles reduce the temperature in the central region of the tray leading to a relatively flat temperature profile. This is possible because the mean free path for $N=10$ particles is approximately the diameter of the experiment. In the hypothetical scenario that the mean free path of the particle is even larger, we might expect some strong influence of the tray diameter on the temperature profile. This would occur since the time between collisions with the wall dominates over interparticle collisions.

In contrast, as the number of particles approaches $N_{c}$ it becomes harder for cold particles reaching the edge of the tray to return to the middle, due to collisions with other particles. This is exacerbated by the fact that the local particle area fraction increases near the boundary reducing the mean free path to approximately the particle diameter. In addition to collisions with a boundary, subsequent collisions with other particles can increase the observed loss. When a particle is reflected from a boundary it has an increased relative velocity with respect to a second particle traveling towards the boundary, making collisions between two particles on average more lossy [26]. However, such mechanisms only contribute significantly when the mean free path is sufficiently short to make such processes probable. When the number of particles approaches $N_{c}$, large differences in the local particle area fraction exist between the center and edge of the tray. This results in differences in the ratio of kinetic to collisional heat transport in these different locations. Consequently, particles at the edge undergo more frequent dissipative collisions resulting in a loss of energy, while those particles in the center remain in a relatively sparsely populated region undergoing fewer collisions and maintaining a higher temperature.

As the number of particles increases still further, local area fraction difference between center and edge reduces [see Fig. 2(a)]. Consequently, collisions result in comparable amounts of loss at both the edge and center, leading to a flatter temperature profile. One therefore expects a peak in $\Delta T / T$ where one has a large number of particles with a large spatial difference in local particle area fraction. This is very similar to the established mechanisms underlying clustering [7].

The effect of cold particles traveling towards the center of the tray for low particle numbers can be observed in experiment by examining the components of the granular temperature. Figure 3 shows the radial $\left\langle v_{r}^{2}\right\rangle(\bullet)$ and tangential granular temperature $\left\langle v_{t}^{2}\right\rangle(\boldsymbol{\square})$ as a function of the radial position for
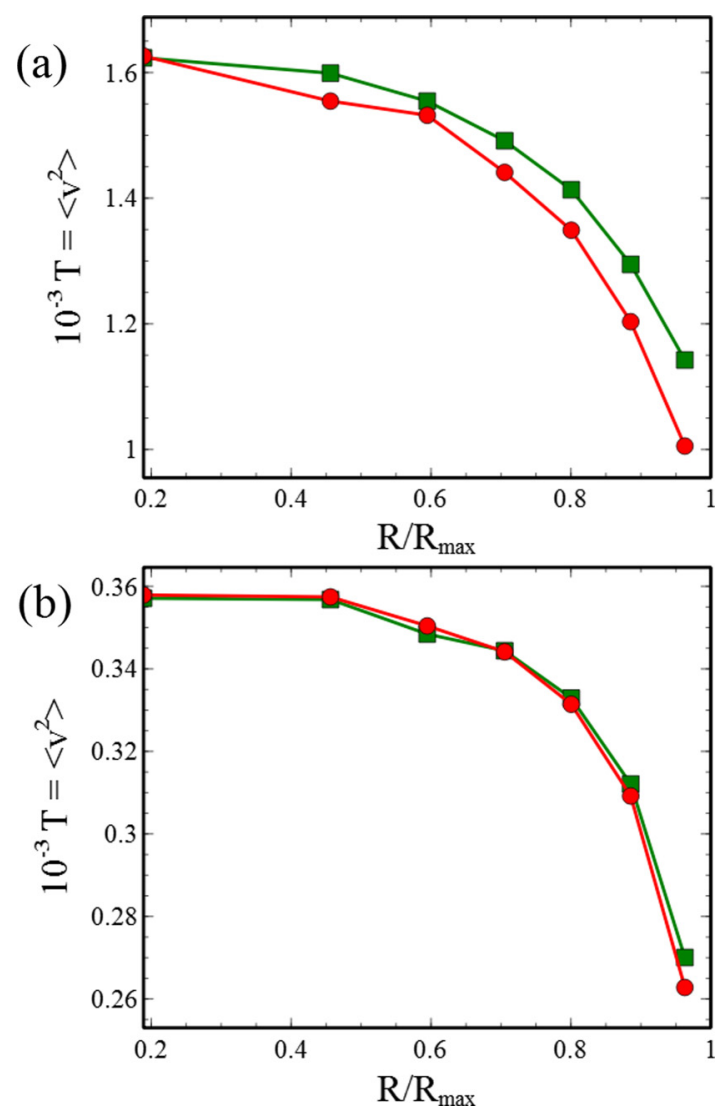

FIG. 3. Anisotropy in the granular temperature. Plots show the radial $\left(\left\langle V_{r}^{2}\right\rangle, \mathbf{0}\right)$ and tangential $\left(\left\langle V_{t}^{2}\right\rangle, \square\right)$ granular temperature at different radial coordinates for (a) $N=20(\varphi=0.025)$ and (b) $N=$ $300(\varphi=0.379)$. At low particle numbers we observe a splitting in the radial and tangential granular temperature profile that persists far from the wall.

(a) $N=20$ and (b) $N=300$ particles. The radial velocity $v_{r}$ was calculated by taking the dot product of the particle velocity with the radial position unit vector (with origin at the center of the circular tray). The tangential velocity was then calculated through subtracting the radial velocity vector from the overall velocity vector $\left(\mathbf{v}_{\mathbf{t}}=\mathbf{v}-\mathbf{v}_{\mathbf{r}}\right)$. For all low particle numbers $(N<100)$ there is a definite splitting between the radial and tangential temperatures. This is more pronounced especially for the lowest numbers of particles.

The radial temperature is always lower than the corresponding tangential temperature. However, for higher particle numbers, with the exception of a small effect in the outermost ring, the radial and tangential temperatures are always equal. In our experiments particles traveling radially inwards will on average have a lower temperature than the particles traveling in the azimuthal direction within that ring. The probability that a particle's current radial temperature is lower than its tangential temperature depends largely on whether the particle is traveling from a region with lower granular temperature without having undergone a direction altering collision. If the mean free path is small the temperature components are indistinguishable due to the large number of collisions that alter the direction of the particle motion. However, if it is sufficiently large to allow a particle to move from one ring 

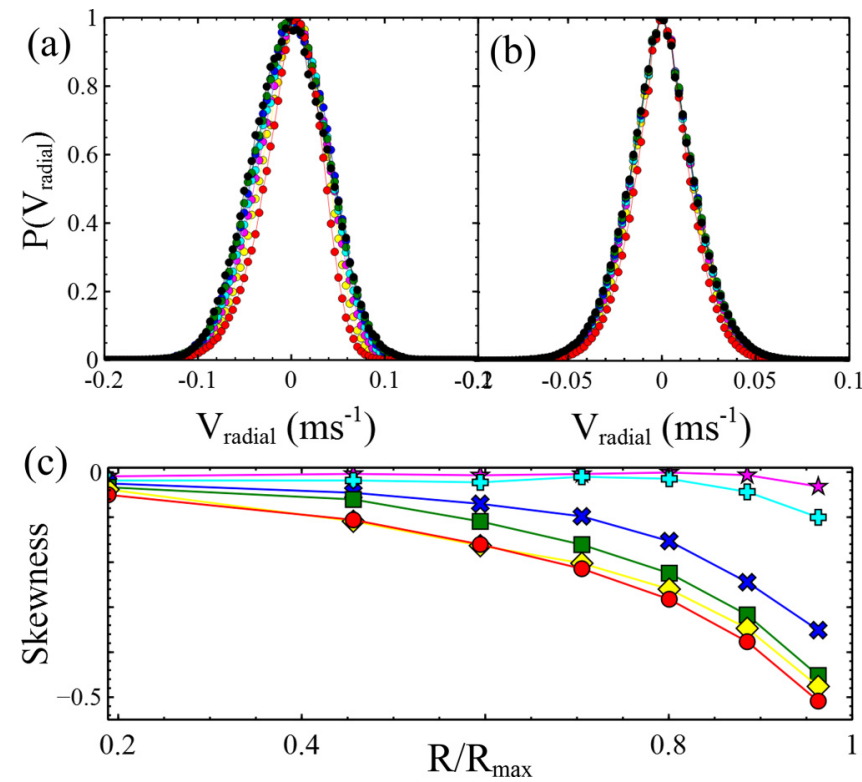

FIG. 4. Skew of radial velocity distributions. Radial velocity distributions for (a) $N=20$, (b) $N=300$ (normalized to 1 for clarity) are plotted for each radial ring $R / R_{\max } \sim 0.19$ (black circle), 0.46 (green circle), 0.59 (blue circle), 0.71 (purple circle), 0.80 (yellow circle), 0.96 (red circle). (c) The skewness of the corresponding velocity distributions for $N=10(\mathbf{0}), 20(\diamond), 50(\square), 100(\aleph), 300$ (や), 500 (艺)。

to another, maintaining the correlation between direction of travel and the radial temperature gradient, it will result in a drop in the measured temperature.

In Fig. 4 we quantify this same behavior more completely by plotting the radial velocity (positive $V_{r}$ indicates particles traveling towards the wall) distributions in each ring for $N=20$ and 300 (where the height of each curve has been normalized to 1 for clarity). For low particle numbers the outermost ring (red circle) displays a strong asymmetry. The distribution implies that most particles travel towards the wall faster than they travel out from the wall. While the loss of energy is unsurprising, what is clearly demonstrated here is that the losses remain preferentially oriented with the radial direction a long way from the boundary. In contrast at high particle densities, sufficient collisions occur to redistribute the loss in all directions, resulting in a far more symmetric distribution [see Fig. 4(b)]. Figure 4(c) shows for all the different particle densities how the skewness of the distributions (characterizing their asymmetry) gradually decreases as one moves further from the wall and as local particle area fraction increases.

In addition to the way in which the distributions quantify the effects of the wall they exhibit a further curiosity. Especially for low particle numbers the tails of the radial distribution [see Fig. 5(a)] at high velocities have a much higher population traveling away from $\left(V_{r}<0\right)$ rather than towards the wall. This is quite unexpected and in contrast to the majority of the central region of the velocity distribution, which in general shows that more particles travel towards the wall faster than they return. Overpopulated tails in particle velocity distributions have been observed in numerous studies concerned with bulk motion [27]. Qualitatively this overpopulation is understood to be due to the fact that the majority of particles
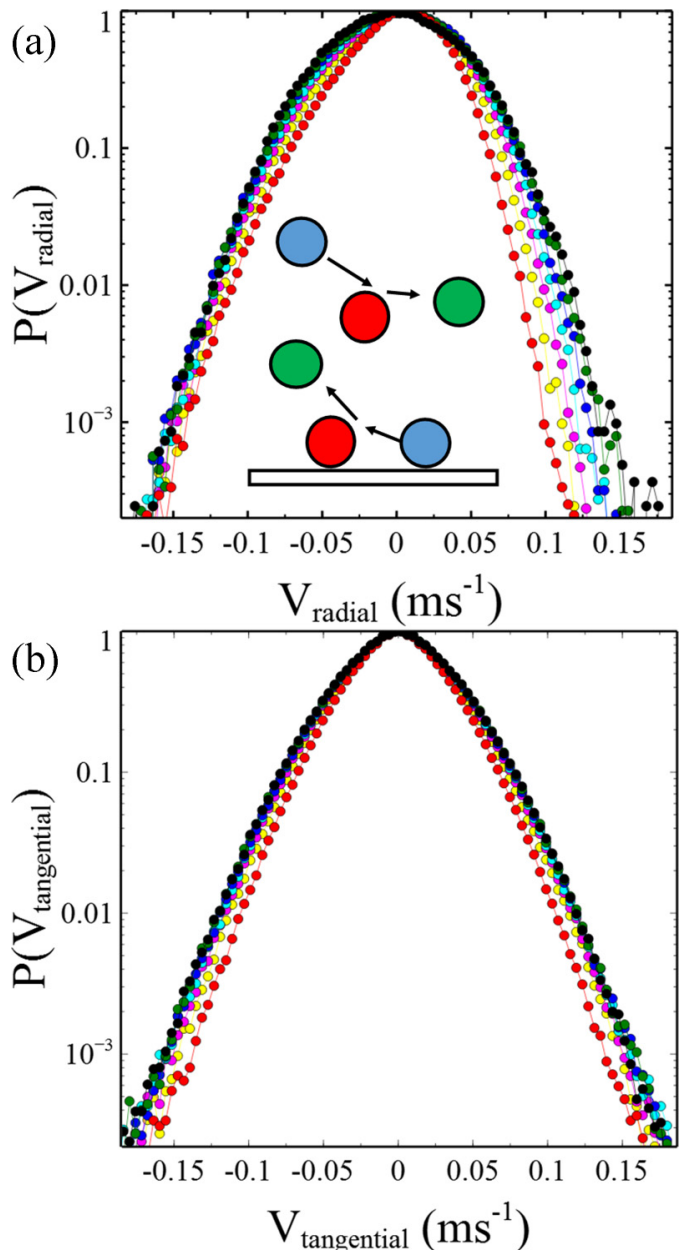

FIG. 5. The tails of the $N=20$ radial and tangential particle velocity distributions. Data are shown for the radial (a) and tangential velocity (b) distributions for each radial ring $R / R_{\text {tot }} \sim 0.19$ (black circle), 0.46 (green circle), 0.59 (blue circle), 0.71 (purple circle), 0.80 (yellow circle), 0.96 (red circle).

undergo loss through collisions resulting in a lower value for the steady-state temperature. However, there are a small number of particles that fail to collide with another particle, over some time interval which is long compared to the mean collision time. These "hot" particles dominate the tails of the distribution resulting in a greater population than would be expected from the steady-state temperature [20]. In our experiments, there is a significantly larger population in the radial distribution of particles traveling away from the wall. Figure 5(a) shows the data in Fig. 4(a) replotted with the $y$ axis on a logarithmic scale to enable the tails of the distribution to be seen more clearly.

Particles traveling radially inwards could be produced by collision of particles traveling radially outwards, with the tray boundary or other particles near to the edge. However, since these collisions result in a reduction in speed one would expect this to result in a distribution whose tails are more heavily populated by particles traveling radially outwards (as is indeed observed for the central portion of the distribution). An alternative way in which high inwards radial velocities can be produced is through rotation of the direction of travel 
of particles traveling in a tangential direction, either through glancing collisions (which result in minimal energy loss) or through the random kicks derived from the vibrating base. It is notable that the population of the tail of the distribution of negative radial velocities is very similar in magnitude to the tangential velocity distributions displayed in Fig. 5(b). Indeed the changes seem to mirror one another for the different radial rings. This might suggest that in the tails of the distribution, the high inwards radial velocities are brought about through changes in the direction of tangential particles.

The radial gradient in the local particle area fraction also introduces an interesting asymmetry which may contribute to enhancing this affect. Since we only consider the tails of the distribution we make the simplifying assumption that high speed particles are those that, for a time long compared with the mean collision time, only participate in glancing collisions (which are low loss) or fail to collide. Consider first particles that undergo glancing collisions. Figure 5(a) illustrates two high speed particles (blue). One has a radial velocity component towards the wall while the other has a radial velocity towards the center. Since the local particle area fraction increases as one moves towards the wall, it is more probable in both cases that glancing collisions will be with the surface of another particle (red) which is facing away from the wall. This results in the new trajectory of the particle (green) being rotated. However, if particles are moving away from (towards) the wall this increases (decreases) the radial component of the velocity. The population of the fastest inwards moving radial particles (the tails of the distribution) is therefore on average increased by transfer of energy from the tangential direction. In contrast, particles moving radially towards the wall, on average experience the opposite effect, resulting in a reduced population in the tails of the distribution. A similar argument can also be made for those particles which fail to collide. The probability of these particles failing to collide (and hence maintaining a high velocity) increases if they random walk towards lower density regions. For those traveling outwards this is achieved by a chance rotating of the direction of travel to be more radial. For those traveling inwards the probability of not colliding is greatest if they travel in a more tangential direction, avoiding regions of high density at larger radii. If particles do not change directions in this way they increase the chance of a lossy collision and hence would no longer be found in the tail of the velocity distributions. This could explain why there is an increased tendency for the tail of the radial velocity distribution to show a larger population traveling towards the center of the tray.

As a whole our results illustrate that as the volume fraction of particles decreases, boundary effects can be observed increasingly far from the wall. While heat transport is governed by collisional dynamics the drop in temperature is only keenly felt a small number of particle diameters from the boundary. Perhaps more importantly for studies of concentrated granular fluids, the dynamics is also isotropic, meaning that boundary effects can usually be safely ignored. In contrast, lower particle densities are complicated and it seems unwise to ever disregard the effects of the boundary unless the size of the experiment is much larger than the one considered here.

\section{CONCLUSION}

In summary, in this paper we have shown that there is a peak in the relative temperature drop $\Delta T / T_{\max }$ for intermediate values of the particle area fraction. This peak coincides with a change in the normalized particle number density profile where the dominant mechanism for heat transfer switches from kinetic to collisional. The radial and tangential velocities of particles show nontrivial additional effects as one approaches the boundary, including a splitting of the radial and tangential granular temperature and a higher population of fast particles moving towards the center rather than towards the edge.

\section{ACKNOWLEDGMENTS}

We thank Dr. James Sharp and Dr. Mike Swift for useful discussions. This work was supported by the Engineering and Physical Sciences Research Council (Grant No. EP/L003651/1), N.S. acknowledges an EPSRC funded studentship; M.S. gratefully acknowledges a Royal Society University Research Fellowship.
[1] G. W. Baxter and J. S. Olafsen, Gaussian statistics in granular gases, Nature 425, 680 (2003).

[2] J. S. Olafsen and J. S. Urbach, Velocity distributions and density fluctuations in a granular gas, Phys. Rev. E 60, R2468(R) (1999).

[3] I. S. Aranson and L. S. Tsimring, Patterns and collective behaviour in granular media: Theoretical concepts, Rev. Mod. Phys. 78, 641 (2006).

[4] P. M. Reis, R. A. Ingale, and M. D. Shattuck, Crystallization of a Quasi-Two-Dimensional Granular Fluid, Phys. Rev. Lett. 96, 258001 (2006).

[5] A. R. Abate and D. J. Durian, Topological persistence and dynamical heterogeneities near jamming, Phys. Rev. E 76, 021306 (2007).

[6] T. P. C. van Noije and M. H. Ernst, Kinetic theory of granular gases, in Granular Gases, edited by T. Pöschel and S. Luding, Lecture Notes in Physics Vol. 564 (Springer, Berlin, 1998).
[7] A. Puglisi, V. Loreto, U. Marini Bettolo Marconi, A. Petri, and A. Vulpani, Clustering and non-Gaussian Behavior in Granular Matter, Phys. Rev. Lett. 81, 3848 (1998).

[8] V. Narayan, S. Ramaswamy, and N. Menon, Long-lived giant number fluctuations in a swarming granular nematic, Science 317, 105 (2007).

[9] D. L. Blair and A. Kudrolli, Velocity correlations in dense granular gases, Phys. Rev. E 64, 050301 (2001).

[10] N. V. Brilliantov and T. Pöschel, Kinetic Theory of Granular Gases (Oxford University Press, Oxford, 2004).

[11] D. Van der Meer, Temperature anisotropy in a driven granular gas, Europhys. Lett. 74, 384 (2006).

[12] R. D. Wildman, T. W. Martin, P. E. Krouskop, J. Talbot, J. M. Huntley, and D. J. Parker, Convection in vibrated annular granular beds, Phys. Rev. E 71, 061301 (2005).

[13] J. S. van Zon and F. C. MacKintosh, Velocity Distributions in Dissipative Granular Gases, Phys. Rev. Lett. 93, 038001 (2004). 
[14] J. A. Perera-Burgos, G. Pérez-Ángel, and Y. NahmadMolinari, Diffusivity and weak clustering in a quasitwo-dimensional granular gas, Phys. Rev. E 82, 051305 (2010).

[15] J. S. Olafsen and J. S. Urbach, Clustering, Order, and Collapse in a Driven Granular Monolayer, Phys. Rev. Lett. 81, 4369 (1998).

[16] J. J. Brey, D. Cubero, and M. J. Ruiz-Montero, High energy tail in the velocity distribution of a granular gas, Phys. Rev. E 59, 1256 (1999).

[17] S. S. Hsiau, L. S. Lu, and C. H. Tai, Experimental investigations of granular temperature in vertical vibrated beds, Powder Technol. 182, 202 (2008).

[18] S. Schollman, Simulation of a two-dimensional shear cell, Phys. Rev. E 59, 889 (1999).

[19] F. V. Reyes and J. S. Urbach, Effect of inelasticity on the phase transitions of a thin vibrated granular layer, Phys. Rev. E 78, 051301 (2008).

[20] J. S. van Zon, J. Kreft, D. I. Goldman, D. Miracle, J. B. Swift, and H. L. Swinney, Crucial role of sidewalls in velocity distributions in quasi-two-dimensional granular gases, Phys. Rev. E 70 , 040301 (2004).
[21] Y. Chen, M. Hou, P. Evesque, Y. Jiang, and M. Liu, Asymmetric velocity distributions in boundary-heating granular gas and a hydrodynamic description, in Powders and Grains 20132013, Sydney, Australia, Proceedings of the 7th International Conference on Micromechanics of Granular Media, AIP Conf. Proc. No. 1542, edited by A. Yu, K. Dong, R. Yang, and S. Luding (AIP, New York, 2013), p. 791.

[22] K. Watanabe, T. Kawasaki, and H. Tanaka, Structural origin of enhanced slow dynamics near a wall in glass-forming systems, Nat. Mater. 10, 512 (2011).

[23] See Supplemental Material at http://link.aps.org/supplemental/ 10.1103/PhysRevE.96.062910 for a video comparing the dynamics of $N=20$ and 300 beads.

[24] B. Andreotti, Y. Forterre, and O. Pouliquen, Granular Media: Between Fluid and Solid (Cambridge University Press, Cambridge, 2013).

[25] S. Meyer, C. Song, Y. Jin, K. Wang, and H. A. Makse, Jamming in 2D packings, Phys. A (Amsterdam, Neth.) 389, 5137 (2010).

[26] T. Pöschel and T. Schwager, Computational Granular Dynamics (Springer, Berlin, 2005).

[27] S. E. Esipov and T. Pöschel, The granular phase diagram, J. Stat. Phys. 86, 1385 (1997). 